\title{
BRD4 and PIN1 gene polymorphisms are associated with high pulse pressure risk in a southeastern Chinese population
}

Jin-jia Qiu ${ }^{1 \dagger}$, Rui-zhi Yang ${ }^{1+}$, Yi-jie Tang ${ }^{2}$, Ying-yi Lin ${ }^{1}$, Hao-jie Xu ${ }^{1}$, Na Zhang ${ }^{1}$, Min Liang ${ }^{1}$, Hong-da Cai ${ }^{1}$, Kai Zeng ${ }^{1 *}$ (D) and Xiao-dan $\mathrm{Wu}^{2^{*}}$

\begin{abstract}
Background: BRD4 and PIN1 have been described to be involved in inflammation and vascular endothelial cell dysfunction, which in turn may increase pulse pressure.

Hypothesis: Genetic mutations within the BRD4 and PIN1 genes could affect the risk of high pulse pressure.

Methods: A total of four single nucleotide polymorphisms (SNPs) (BRD4: rs4808278; PIN1: rs2233678, rs2287838, and rs2233682) were genotyped in a cohort of 666 hypertensive patients and 232 normotensive controls with Chinese Han origin. Generalized multifactor dimensionality reduction (GMDR) was used to screen the best interaction combination among the four SNPs within the BRD4 and PIN1 genes and diabetes. Logistic regression analysis was performed to calculate the odds ratio (ORs) (95\% confidence interval (CI)) for the association between the four SNPs.

Results: Adjusted for age, weight, waist circumference, drinking, smoking, hypertension, and diabetes, high pulse pressure risk was significantly higher for carriers with the rs4808278-TT genotype in BRD4 than those with wild genotypes (OR: $0.400,95 \% \mathrm{Cl}: 0.217-0.737, P^{*}<0.05$ ). However, we did not find any significant association of rs 2233678 , rs2287838, and rs2233682 in PIN1 with high pulse pressure susceptibility after covariate adjustment. GMDR analysis indicated a significant three-locus model $(P=0.0107)$ involving rs4808278, rs2233678, and diabetes, the cross-validation consistency of the three-locus models was $9 / 10$, and the testing accuracy was $57.47 \%$.
\end{abstract}

Conclusions: Genetic mutations within BRD4 (rs4808278) could affect the susceptibility to high pulse pressure in a southeastern Chinese population.

Keywords: High pulse pressure, BRD4, Single nucleotide polymorphisms

\section{Background}

Pulse pressure (PP) refers to the difference between systolic and diastolic blood pressure, of which the normal range is $30-50 \mathrm{mmHg}$ [1]. As an independent risk factor

\footnotetext{
*Correspondence: fymzk6822@163.com; wxiaodan@sina.com ${ }^{\dagger}$ Jin-jia Qiu and Rui-zhi Yang have contributed equally to this study ${ }^{1}$ Department of Anesthesiology, The First Affiliated Hospital, Fujian Medical University, Fuzhou 350005, Fujian, China

${ }^{2}$ Department of Anesthesiology, Fujian Provincial Hospital, Fujian Provincial Clinical Medical College, Fujian Medical University, Fuzhou 350001, Fujian, China
}

of cardiovascular disease, $\mathrm{PP}$ is attracting more attention from doctors, and its forecast value may be larger than systolic blood pressure (SBP) or diastolic blood pressure (DBP) [2]. Hypertension and cardiocerebrovascular diseases caused by vascular diseases are often accompanied by elevated PP [3-5]. Therefore, a comprehensive understanding of the molecular basis of high PP is crucial for the development of potential therapeutic strategies.

The decrease in vascular elasticity is an important factor for the increase in pulse differential pressure and may result from inflammation and vascular endothelial 
cell dysfunction. Many novel loci related to blood pressure (BP) adjustment have been identified by researchers. However, it is suggested that there is a possible presence of allelic heterogeneity in BP regulation between Europeans and Asians, which provide new mechanistic insights into cardiovascular disease [6]. A previous study has reported that BRD4 and PIN1 are involved in inflammation and vascular endothelial cell dysfunction. As a transcription regulatory factor, $B R D 4$ may lead to extracellular calcium deposition, and BRD4 may cooperate with seven specific transcription factors to promote calcification [7]. BRD4 silencing results in a significant reduction in inflammatory cytokine expression [8]. Multiple studies have shown that PIN1 binds to endothelial nitric oxide (NO) synthase and enables dephosphorylation of serine, which increases NO production and endothelium-dependent dilation, leading to BP maintenance [9]. Inhibiting $B r d 4$ and $\operatorname{Pin} 1$ expression alleviates enhancermediated inflammatory transcription and atherosclerosis, which indicates that BRD4 and PIN1 play a vital role in the development of vascular inflammation and atherosclerosis [10-13]. Therefore, it is possible that variants in BRD4 and PIN1 genes may lead to decreased vascular elasticity by destroying endothelial cells and causing atherosclerosis.

The aim of this study was to investigate the association between BRD4 and PIN1 gene polymorphisms and high $\mathrm{PP}$ in a Chinese population.

\section{Methods Subjects}

Han Chinese who visited the Fujian Medical University Affiliated with the First Hospital (from September 2014 to December 2016) were potential study subjects for this research. Patients with renal failure, severe heart disease (e.g. myocardial infarction, congenital heart disease, serious arrhythmia, valvulopathy or other atherosclerotic lesions), gestation, severe psychiatric disorder, cancer, peripheral vascular disease, severe anemia, severe metabolic disease, or infectious disease were excluded. Therefore, 232 healthy control subjects $(\mathrm{SBP}<140 \mathrm{~mm}$ $\mathrm{Hg}$ and $\mathrm{DBP}<90 \mathrm{~mm} \mathrm{Hg}$ ) who were not taking antihypertensive medication and 666 hypertensive patients ( $\mathrm{SBP} \geq 140 \mathrm{~mm} \mathrm{Hg}$ or $\mathrm{DBP} \geq 90 \mathrm{~mm} \mathrm{Hg}$ ) were selected for the study. Also, BP was measured three times using a calibrated mercury sphygmomanometer by certified examiners with at least $10 \mathrm{~min}$ interval and taken the average of three readings. The difference between systolic and diastolic blood pressure is PP. The aims of the study were carefully explained to all participants, who signed an informed written consent. The project complied with the Declaration of Helsinki guidelines and was approved by the Institute's Human Research and Ethics Committees.

\section{Single nucleotide polymorphism (SNP) selection and genotyping}

SNPs associated with risk factors for high pulse differential pressure and their minor allele frequencies are greater than 5\% in the 1000 Genomes Project (https://www.inter nationalgenome.org/). We used DNA isolation kits (Taijing Biological Technology [Xiamen], China) to extract genomic DNA from blood samples collected in anticoagulant ethylene diamine tetraacetic acid(EDTA) tubes and stored at $-20^{\circ} \mathrm{C}$. Five SNPs (rs4808278, rs2233678, rs2233679, rs2287838, and rs2233682) were genotyped using the polymerase chain reaction ligase detection reaction (PCR-LDR) method with five TaqMan genotyping assays on a Light Cycler 480 Instrument (Roche Diagnostics, Basel, Switzerland). Taqman probes and primers for the BRD4 and PIN1 genes were synthesized by Shanghai Generay Biotech (see Additional file 2: Table S1, in the online version of this article). PCR amplification was carried out in a PTC-200 MJ Research Peltier Thermal Cycler (Bio-Rad Lab, Massachusetts, USA). Genotyping of the five examined SNPs was performed as previously described [14].

\section{Statistical analysis}

SPSS v.25.0 was used for statistical analyses. Means and standard deviations (SDs) were calculated for normally distributed continuous variables, and percentages were calculated for categorical variables. The Student's t-test was used to compare normally distributed numeric variables between two groups, otherwise a non-parametric test was used. SHESIS software (https://www.nhgg.org/ analysis/) was used to calculate Hardy-Weinberg equilibrium (HWE), genotype/allele frequencies, and haplotype analysis $[15,16]$. To evaluate the association between SNPs and high PP, logistic regression analysis was used to examine the odds ratios (ORs) and 95\% confidence intervals (CIs). Generalized multifactor dimensionality reduction (GMDR) was used to detect gene-gene and gene-environment interactions. $P$ values reported were two-tailed and those less than 0.05 were considered statistically significant. The analysis results were also processed using an online calculator for false discovery rate (FDR) correction (https://www.sdmproject.com/utili ties/?show=FDR).

\section{Results}

\section{Clinical characteristics of the study population}

We recorded baseline characteristics including anthropometric index and clinical biomarkers between patients with high (243 males, 217 females) and normal (275 
males, 163 females) PP. As shown in Table 1, there was no statistical difference in weight $(P>0.05)$ between the two groups. In addition, cases and controls were comparable with respect to height, drinking, and smoking $(P<0.05)$. Older subjects with higher levels of body mass index (BMI), waist circumference, SBP, DBP, obesity, diabetes, and smoking had a high risk of high PP $(P<0.001)$.

\section{Associations between BRD4 and PIN1 SNPs and high PP}

Of the five SNPs in BRD4 and PIN1 analyzed in this study, one SNP (rs2233679) was excluded because of significant deviation from HWE $(P<0.05)$, whereas the other SNPs satisfied the HWE in the control groups. Allele frequencies and basic information for all SNPs are shown in Table 2 and Additional file 1. The frequency of rs4808278 genotypes $(P=0.046878)$ was significantly different between normal PP and high PP groups. No significant difference was found in the genotype distribution of rs2233678, rs2233682, and rs2287838 between the two groups.

Three risk prediction models (additive model, dominant model, and recessive model) were used to evaluate the association between these gene polymorphisms and high PP risk. The data show that rs4808278 is markedly associated with high PP under the additive and recessive models. The adjusted $P$ value was further increased after adjusting for age, weight, waist circumference, drinking, smoking, hypertension, and diabetes.
As a result, carriers of the rs4808278-A allele have a higher risk of developing high PP compared those carrying the rs4808278-TT genotype after adjustment for confounding factors $(\mathrm{OR}=0.400$; 95\% CI: $0.217-$ $0.737 ; P=0.003$ ). No significance was observed for the remaining polymorphisms under all models (see Additional file 2: Table S2).

\section{Haplotype analyses of BRD4 and PIN1 SNPs}

SHESIS software was used to perform haplotype analyses. Compared to the control group, no significant difference was found in haplotypes of the PIN1 gene in the case group (see Additional file 2: Table S3).

\section{GMDR analysis of BRD4 and PIN1 SNPs and high PP risk}

The GMDR model was used to investigate the best interaction combination among the four SNPs and smoking on high PP risk. Table 3 summarizes the results obtained from GMDR analysis for gene-gene and gene-diabetes interactions. The best three-locus models for gene-diabetes interaction $(P=0.0107)$ were rs4808278, rs2287838, and diabetes after adjusting for the covariates (age, sex, smoking, drinking and BMI), of which the cross-validation consistency was 9/10. However, we did not find a significant any-locus model among the four SNPs.

Table 1 Comparison of baseline clinical characteristics among groups of normal pulse pressure and high pulse pressure

\begin{tabular}{|c|c|c|c|}
\hline Indicators & Normal pulse pressure group $(n=438)$ & High pulse pressure group $(n=460)$ & $P$ value \\
\hline \multicolumn{4}{|l|}{ Sex } \\
\hline Males & 275 & 243 & 0.004 \\
\hline Females & 163 & 217 & \\
\hline Age (year) & $56.94 \pm 13.38$ & $65.01 \pm 11.36$ & $<0.001$ \\
\hline Height (cm) & $164.70 \pm 7.51$ & $163.36 \pm 7.92$ & 0.008 \\
\hline Weight (kg) & $64.93 \pm 11.58$ & $66.34 \pm 11.40$ & 0.065 \\
\hline $\mathrm{BMI}\left(\mathrm{kg} / \mathrm{m}^{2}\right)$ & $23.88 \pm 3.67$ & $24.79 \pm 3.42$ & $<0.001$ \\
\hline Waist circumference (cm) & $84.87 \pm 10.23$ & $88.30 \pm 9.66$ & $<0.001$ \\
\hline $\mathrm{SBP}(\mathrm{mmHg})$ & $138.05 \pm 19.64$ & $174.93 \pm 23.83$ & $<0.001$ \\
\hline $\mathrm{DBP}(\mathrm{mmHg})$ & $87.69 \pm 17.56$ & $93.28 \pm 17.31$ & $<0.001$ \\
\hline $\mathrm{PP}(\mathrm{mmHg})$ & $50.36 \pm 8.14$ & $81.65 \pm 16.16$ & $<0.001$ \\
\hline Obesity (\%) & $156(35.62)$ & $244(53.04)$ & $<0.001$ \\
\hline Diabetes (\%) & $103(23.52)$ & $198(43.04)$ & $<0.001$ \\
\hline \multicolumn{4}{|l|}{ Smoking (\%) } \\
\hline Smoker & 275 (62.79) & $324(70.43)$ & $<0.001$ \\
\hline Former-smokers & 118 (26.94) & $71(15.44)$ & \\
\hline Nonsmoker & $45(10.27)$ & $65(14.13)$ & \\
\hline Drinking (\%) & $63(14.38)$ & $38(8.26)$ & 0.005 \\
\hline
\end{tabular}

Data are expressed as mean and standard deviation (SD). $P$ values were corrected using FDR

$B M I$ body mass index, $S B P$ systolic blood pressure, $D B P$ diastolic blood pressure, $P P$ pulse pressure, $D R$ false discovery rate 
Table 2 Genotype and allele frequencies of four single nucleotide polymorphisms in the BRD4 and PIN1 genes in normal and high pulse pressure groups

\begin{tabular}{|c|c|c|c|c|}
\hline \multirow[t]{2}{*}{ SNPs } & \multirow[t]{2}{*}{ Genotypes and alleles } & \multicolumn{2}{|c|}{ Frequencies $\mathrm{N}(\%)$} & \multirow[t]{2}{*}{$P$ value } \\
\hline & & Case $(n=460)$ & Control $(n=438)$ & \\
\hline & AA & $250(0.543)$ & $240(0.548)$ & 0.046878 \\
\hline BRD4 & AT & $164(0.357)$ & $173(0.395)$ & \\
\hline \multirow[t]{3}{*}{ rs4808278 } & $\mathrm{TT}$ & $46(0.100)$ & $25(0.057)$ & \\
\hline & Allele, T (\%) & $256(0.278)$ & $223(0.255)$ & 0.256363 \\
\hline & GG & $443(0.963)$ & $418(0.954)$ & 0.511810 \\
\hline PIN1 & $C G$ & $17(0.037)$ & $20(0.046)$ & \\
\hline \multirow[t]{3}{*}{ rs2233678 } & $\mathrm{CC}$ & $0(0)$ & $0(0)$ & \\
\hline & Allele, C (\%) & $17(0.018)$ & $20(0.023)$ & 0.516271 \\
\hline & GG & $438(0.952)$ & $422(0.963)$ & 0.400651 \\
\hline PIN1 & $A G$ & $22(0.048)$ & $16(0.037)$ & \\
\hline \multirow[t]{3}{*}{ rs2233682 } & $\mathrm{AA}$ & $0(0)$ & $0(0)$ & \\
\hline & Allele, A(\%) & $22(0.024)$ & $16(0.018)$ & 0.405751 \\
\hline & CC & $225(0.489)$ & $232(0.530)$ & 0.476216 \\
\hline PIN1 & $\mathrm{CT}$ & $184(0.400)$ & $162(0.370)$ & \\
\hline \multirow[t]{2}{*}{ rs2287838 } & $\mathrm{TT}$ & $51(0.111)$ & $44(0.100)$ & \\
\hline & Allele, T (\%) & $286(0.311)$ & $250(0.285)$ & 0.238116 \\
\hline
\end{tabular}

A chi-squared test was used to calculate the $P$ values

Table 3 GMDR analysis on the best SNP-SNP and gene-diabetes interaction models

\begin{tabular}{|c|c|c|c|c|}
\hline Locus no & Best combination & $\begin{array}{l}\text { Cross-validation } \\
\text { consistency }\end{array}$ & Testing accuracy & $P$ values \\
\hline \multicolumn{5}{|c|}{ SNP-SNP interactions ${ }^{\mathrm{a}}$} \\
\hline 2 & rs2233682, rs4808278 & $8 / 10$ & 0.5093 & 0.1719 \\
\hline 3 & rs 2233678, rs 2233682, rs 4808278 & $7 / 10$ & 0.4991 & 0.1719 \\
\hline \multicolumn{5}{|c|}{ Gene-diabetes interactions $^{\mathrm{b}}$} \\
\hline 2 & rs4808278, diabetes & $10 / 10$ & 0.5783 & 0.0107 \\
\hline 3 & rs 2233678 , rs4808278, diabetes & $9 / 10$ & 0.5747 & 0.0107 \\
\hline
\end{tabular}

GMDR generalized multifactor dimensionality reduction, SNP single nucleotide polymorphism, BMI body mass index

a Adjusted for age, sex, smoking, drinking, BMI and diabetes

b Adjusted for age, sex, smoking, drinking and BMI

\section{Mapping of associated SNPs at expression quantitative trait loci (eQTLs)}

Rs4808278 was annotated using the RegulomeDB, a database that identifies DNA features and regulatory elements in non-coding regions of the human genome. Known and predicted regulatory DNA elements include DNase hypersensitivity regions, transcription factor (TF) binding sites, and promoter regions that have been biochemically characterized to regulate transcription [17]. Therefore, we investigated the function of rs4808278 in gene expression regulation by exploring the RegulomeDB. The RegulomeDB score of rs 4808278 in BRD4 was 4, suggesting that rs 4808278 may be part of a TF binding site or DNase hypersensitive region.

\section{Discussion}

Scholars are paying more attention to heredity as an important factor in high pulse differential pressure [18, 19]. To investigate a potential association between five promising polymorphisms in the BRD4 and PIN1 genes and high PP risk in a southeastern Chinese population, we designed this pilot case-control study that includes 666 hypertensive patients and 232 normotensive subjects according to exclusion and inclusion criteria. After excluding one SNP (rs2233679) that was not in HWE, we analyzed the remaining four SNPs. There was no statistically significant correlation between the four SNPs and hypertension risk, but we unexpectedly found that rs4808278 in BRD4 was associated with high PP risk, 
suggesting that $B R D 4$ may play an important role in high PP progression. This discovery may provide a new therapeutic target gene.

$B R D 4$, a member of the bromo and extra-terminal family, plays a role in various vascular pathologies. Knocking down $\mathrm{Brd} 4$ with siRNA mitigates neointima formation and vascular remodeling $[20,21]$. The protein also regulates vascular endothelial growth factor (VEGF)-induced angiogenesis by activating phosphorylation of vascular endothelial growth factor receptor 2 (VEGFR2) and P21 activated kinase 1 (PAK1) [22, 23]. To some extent, these pathologies are related to changes in vascular elasticity, which may affect PP variation. Through binding with acetylated RelA, BRD4 enhances activation of NF- $\kappa B$ signaling to induce natriuretic peptide precursor $\mathrm{A}(N P P A)$ and natriuretic peptide precursor $\mathrm{B}(N P P B)$ transcription [24]. Our previous research also found that a SNP in the NPPB gene is associated with high PP [14]. BRD4 plays a critical role in propagating inflammation and promoting vascular calcification in particular $[7,8]$. More importantly, $B R D 4$, a transcriptional regulator, mediates inflammatory transcription, atherogenic endothelial response, and atherosclerosis [10, 11]. Vascular damage, an antecedent to atherosclerosis, leads to an increase in PP and to atherosclerosis, which in turn results in large-vessel stiffening and increased wave reflection. While it is not clear what is the incipient event in this cycle, it is clear that, once initiated, a vicious cycle promoting disease progression ensues. The association analysis of PP with different genotypes show that rs4808278 genotype TT carriers have a lower risk than AT and AA genotypes carriers to have higher PP $(\mathrm{OR}=0.405 ; 95 \% \mathrm{CI}: 0.220-0.745 ; P=0.004)$, which indicates that the $\mathrm{T}$ allele has a protective effect. Although rs4808278 is located in an intron of $B R D 4$, the RegulomeDB score of rs 4808278 in $B R D 4$ is 4, which suggests that rs 4808278 may be part of a TF binding site or DNase hypersensitive region. This suggests that rs 4808278 may affect PP by regulating BRD4 expression. Few studies have reported on SNPs in BRD4 and high PP. Our results may provide new research directions on high $P$.

High PP, diabetes mellitus, and atherosclerosis are closely related, and PP is considered an independent predictor of new-onset diabetes $[25,26]$. Therefore, diabetes and the four SNPs were included in the GMDR analysis. We found a significant three-locus model $(P=0.0010)$ involving rs4808278, rs2233678, and diabetes after adjusting the covariates. The detailed mechanism underlying this interaction is unknown, but all of them are associated with vascular elasticity, which may be the basis for high PP.

There are several limitations in the present work. Firstly, only five SNPs were studied. Secondly, this is a retrospective case-control study. Our original intention was to look for the relationship between SNPs in BRD4/PIN1 and hypertension, but we did not find any association. Thirdly, the presence of atherosclerosis, an important factor causing an increase in PP, was not recorded in the patients.

\section{Conclusion}

In conclusion, our results indicate that genetic mutations within the BRD4 gene (rs4808278) could affect the susceptibility to high PP in a southeastern Chinese population.

\section{Supplementary information}

Supplementary information accompanies this paper at https://doi. org/10.1186/s12872-020-01757-x.

\section{Additional file 1: SNPs in this research.}

Additional file 2: Table S1 Description and primer sequences of the single nucleotide polymorphisms (SNPs) used for polymerase chain reaction (PCR) analysis. Table S2 Prediction of high pulse pressure risk of four single nucleotide polymorphisms in the BRD4 and PIN1 genes under additive, dominant, and recessive models. Table S3 Allele combination analysis of single nucleotide polymorphisms in the PIN1 gene and risk prediction for high pulse pressure.

\section{Abbreviations}

PP: Pulse pressure; SBP: Systolic blood pressure; DBP: Diastolic blood pressure; BP: Blood pressure; NO: Nitric oxide; SNP: Single nucleotide polymorphism; EDTA: Ethylene diamine tetraacetic acid; PCR-LDR: Polymerase chain reaction ligase detection reaction; SDs: Standard deviations; HWE: Hardy-Weinberg equilibrium; ORs: Odds ratios; Cls: Confidence intervals; GMDR: Generalized multifactor dimensionality reduction; FDR: False discovery rate; BMI: Body mass index; eQTLs: Expression quantitative trait loci; TF: Transcription factor; VEGF: Vascular endothelial growth factor; VEGFR2: Vascular endothelial growth factor receptor 2; PAK1: P21 activated kinase 1; NPPA: Natriuretic peptide precursor A; NPPB: Natriuretic peptide precursor $B$.

\section{Acknowledgements}

Not Applicable.

\section{Authors' contributions}

$X W$ and $K Z$ conceived and designed the study. JQ, RY, YT, HX, NZ, ML and $\mathrm{HC}$ performed the experiments and statistical analysis. JQ and RY wrote the paper. $\mathrm{HC}$ and $\mathrm{KZ}$ reviewed and edited the manuscript. All authors read and approved the manuscript.

\section{Funding}

This work was supported by National Natural Science Foundation of China $(81570219,81670264)$ and Excellent Youth Foundation of Fujian Provincial Hospital, China (2017B017). The funding bodies played no role in the design of the study and collection, analysis, and interpretation of data and in writing the manuscript.

\section{Availability of data and materials}

The datasets generated and analysed during the current study are included within the article.

\section{Ethics approval and consent to participate}

This study was approved by the Ethics Review Committee for Biomedical Research of Fujian Medical University (No.2013-24). And all participants signed the informed consent. 


\section{Consent for publication}

Not applicable.

\section{Competing interests}

The authors declare that they have no competing interests.

Received: 25 February 2020 Accepted: 26 October 2020 Published online: 04 November 2020

\section{References}

1. Dart AM, Kingwell BA. Pulse pressure-a review of mechanisms and clinical relevance. J Am Coll Cardiol. 2001;37(4):975-84.

2. Safar M. Arterial stiffness as a risk factor for clinical hypertension. Nat Rev Cardiol. 2018;15(2):97-105.

3. Van Bortel L, Struijker-Boudier H, Safar M. Pulse pressure, arterial stiffness, and drug treatment of hypertension. Hypertension. 2001;38(4):914-21.

4. Pancholy S, Shantha G, Patel T, Sobotka P, Kandzari D. Meta-analysis of the effect of renal denervation on blood pressure and pulse pressure in patients with resistant systemic hypertension. Am J Cardiol. 2014;114(6):856-61.

5. Jaźwiec P, Gać P, Poręba M, Sobieszczańska M, Mazur G, Poręba R. The volume of the carotid bodies and blood pressure variability and pulse pressure in patients with essential hypertension. Clin Radiol. 2016;71(6):616. e617-616.e613.

6. Wang Y, Wang JG. Genome-wide association studies of hypertension and several other cardiovascular diseases. Pulse (Basel). 2019;6(3-4):169-86.

7. Gilham D, Tsujikawa L, Sarsons C, Halliday C, Wasiak S, Stotz S, Jahagirdar $\mathrm{R}$, Sweeney M, Johansson J, Wong N, et al. Apabetalone downregulates factors and pathways associated with vascular calcification. Atherosclerosis. 2019;280:75-84

8. Liong S, Barker G, Lappas M. Bromodomain protein BRD4 is increased in human placentas from women with early-onset preeclampsia. Reproduction. 2018;155(6):573-82.

9. Chiasson V, Munshi N, Chatterjee P, Young K, Mitchell B. Pin1 deficiency causes endothelial dysfunction and hypertension. Hypertension. 2011;58(3):431-8.

10. McLure K, Gesner E, Tsujikawa L, Kharenko O, Attwell S, Campeau E, Wasiak S, Stein A, White A, Fontano E, et al. RVX-208, an inducer of ApoA-I in humans, is a BET bromodomain antagonist. PLOS ONE. 2013:8(12):e83190.

11. Brown J, Lin C, Duan Q, Griffin G, Federation A, Paranal R, Bair S, Newton G, Lichtman A, Kung A, et al. NF-kB directs dynamic super enhancer formation in inflammation and atherogenesis. Mol Cell. 2014;56(2):219-31.

12. Lv L, Ye M, Duan R, Yuan K, Chen J, Liang W, Zhou Z, Zhang L. Downregulation of Pin 1 in human atherosclerosis and its association with vascular smooth muscle cell senescence. J Vasc Surg. 2018:68(3):873-883.e875.

13. Liu M, Yu P, Jiang H, Yang X, Zhao J, Zou Y, Ge J. The Essential role of Pin1 via NF-KB signaling in vascular inflammation and atherosclerosis in ApoE mice. Int J Mol Sci. 2017;18(3):644

14. Zeng K, Wu X, Cai H, Gao Y, Li G, Liu Q, Gao F, Chen J, Lin C. Correlation between the NPPB gene promoter c.-1298 G/T polymorphism site and pulse pressure in the Chinese Han population. Genet Mol Res. 2014;13(2):3265-74.

15. Li Z, Zhang Z, He Z, Tang W, Li T, Zeng Z, He L, Shi Y. A partition-ligationcombination-subdivision EM algorithm for haplotype inference with multiallelic markers: update of the SHEsis (https://analysis.bio-x.cn/). Cell Res. 2009;19(4):519-23.

16. Shi Y, He L. SHEsis, a powerful software platform for analyses of linkage disequilibrium, haplotype construction, and genetic association at polymorphism loci. Cell Res. 2005;15(2):97-8.

17. Boyle A, Hong E, Hariharan M, Cheng Y, Schaub M, Kasowski M, Karczewski K, Park J, Hitz B, Weng S, et al. Annotation of functional variation in personal genomes using RegulomeDB. Genome Res. 2012;22(9):1790-7.

18. Stokes L, Scurrah K, Ellis J, Cromer B, Skarratt K, Gu B, Harrap S, Wiley J. A loss-of-function polymorphism in the human $\mathrm{P} 2 \mathrm{X} 4$ receptor is associated with increased pulse pressure. Hypertension. 2011;58(6):1086-92.

19. Engelen L, Ferreira I, Brouwers O, Henry RM, Dekker JM, Nijpels G, Heine RJ, van Greevenbroek MM, van der Kallen CJ, Blaak EE, et al. Polymorphisms in glyoxalase 1 gene are not associated with vascular complications: the Hoorn and CoDAM studies. J Hypertens. 2009;27(7):1399-403.

20. Wang B, Zhang M, Takayama T, Shi X, Roenneburg D, Kent K, Guo L. BET bromodomain blockade mitigates intimal hyperplasia in rat carotid arteries. EBioMedicine. 2015;2(11):1650-61.

21. Meloche J, Lampron M, Nadeau V, Maltais M, Potus F, Lambert C, Tremblay E, Vitry G, Breuils-Bonnet S, Boucherat O, et al. Implication of inflammation and epigenetic readers in coronary artery remodeling in patients with pulmonary arterial hypertension. Arterioscler Thromb Vasc Biol. 2017;37(8):1513-23.

22. Chen J, Fu Y, Day D, Sun Y, Wang S, Liang X, Gu F, Zhang F, Stevens S, Zhou $P$, et al. VEGF amplifies transcription through ETS1 acetylation to enable angiogenesis. Nat Commun. 2017;8(1):383.

23. Huang M, Qiu Q, Xiao Y, Zeng S, Zhan M, Shi M, Zou Y, Ye Y, Liang L, Yang $X$, et al. BET bromodomain suppression inhibits VEGF-induced angiogenesis and vascular permeability by blocking VEGFR2-mediated activation of PAK1 and eNOS. Sci Rep. 2016;6:23770.

24. Sun Y, Xie Y, Du L, Sun J, Liu Z. Inhibition of BRD4 attenuates cardiomyocyte apoptosis via NF-kB pathway in a rat model of myocardial infarction. Cardiovasc Ther. 2018;36(2):e12320.

25. Yasuno S, Ueshima K, Oba K, Fujimoto A, Hirata M, Ogihara T, Saruta T, Nakao K. Is pulse pressure a predictor of new-onset diabetes in high-risk hypertensive patients?: A subanalysis of the Candesartan Antihypertensive Survival Evaluation in Japan (CASE-J) trial. Diabetes Care. 2010:33(5):1122-7.

26. Zhang L, Wang B, Wang C, Li L, Ren Y, Zhang H, Yang X, Zhao Y, Han C, Zhou J, et al. High pulse pressure is related to risk of type 2 diabetes mellitus in Chinese middle-aged females. Int J Cardiol. 2016;220:467-71.

\section{Publisher's Note}

Springer Nature remains neutral with regard to jurisdictional claims in published maps and institutional affiliations.

Ready to submit your research? Choose BMC and benefit from

- fast, convenient online submission

- thorough peer review by experienced researchers in your field

- rapid publication on acceptance

- support for research data, including large and complex data types

- gold Open Access which fosters wider collaboration and increased citations

- maximum visibility for your research: over 100M website views per year

At BMC, research is always in progress.

Learn more biomedcentral.com/submissions 